\title{
Earthquake Seismic Moment, Rupture Radius and Stress-drop from P-wave Displacement Amplitude vs Time Curves
}

This paper was downloaded from TechRxiv (https://www.techrxiv.org).

\section{LICENSE}

CC BY 4.0

SUBMISSION DATE / POSTED DATE

$13-07-2021 / 15-07-2021$

\section{CITATION}

zollo, aldo; nazeri, sahar; Colombelli, Simona (2021): Earthquake Seismic Moment, Rupture Radius and Stress-drop from P-wave Displacement Amplitude vs Time Curves. TechRxiv. Preprint. https://doi.org/10.36227/techrxiv.14959260.v1

$\mathrm{DOI}$

10.36227/techrxiv.14959260.v1 


\title{
Earthquake Seismic Moment, Rupture Radius and Stress-drop from P-wave Displacement Amplitude vs Time Curves
}

\author{
Aldo Zollo, Sahar Nazeri, Simona Colombelli
}

\begin{abstract}
The reliable determination of earthquake source parameters is a relevant task of seismological investigations which ground nowadays on high quality seismic waveforms collected by near-source dense arrays of ground motion sensors. Here we propose a parametric modelling technique which analyzes the time-domain $\mathbf{P}$-wave signal recorded in the near-source range of small-to-large size earthquakes. Assuming a triangular momentrate function and a uniform speed, circular rupture model, we develop the equations to estimate the seismic moment, rupture radius and stress-drop from the corner-time and plateau level of the average logarithm of the $P$-wave displacement vs time curves (LPDT). The constant- $Q$, anelastic attenuation effect is accounted by a post-processing procedure that evaluates the $Q$-unperturbed moment-rate triangular shape.

The methodology has been validated through the application to the acceleration records of the 2016-2017 Central Italy and 2007-2019 Japan earthquake sequences covering a wide moment magnitude range (Mw $2.5-6.5$ ) and recording distance $<100 \mathrm{~km}$. After correcting for the anelastic attenuation function, the estimated average stress-drop and the confidence interval $(\langle\Delta \sigma\rangle=0.60(0.42$ 0.87) MPa and $\langle\Delta \sigma\rangle=1.53(1.01-2.31)$ for crustal and subcrustal events of Japan and $\langle\Delta \sigma\rangle=0.36(0.30-0.44)$ MPa for Central Italy) show, for both regions, a self-similar, constant stress-drop scaling of the rupture duration/radius with seismic moment. The smaller sensitivity of the spatially averaged, time-varying peak displacement amplitude to the radiation from localized high slip patch on the fracture surface, could explain the retrieved smaller average stress-drops for sub-crustal earthquakes in Japan and $M>5.5$ events in Central Italy relative to previous estimates using spectral methods.
\end{abstract}

Index Terms - Earthquake source parameters, P-wave displacement signals, parametric modelling technique, timedomain, near-source, far-field, source time function.

\section{INTRODUCTION}

arthquake rupture is a complex phenomenon whose details are investigated through the analysis and modelling of the radiated seismic wave field recorded at the Earth surface. However, the estimation of the earthquake rupture parameters (i.e., stress conditions, fault friction, slip-weakening, etc.) from seismograms is a difficult and uncertain task since seismic waves travel from the deep interior to the Earth surface and

This work has been funded by several projects as: (1) Project "SERA Seismology and Earthquake Engineering Research, Infrastructure Alliance for Europe" - Grant Agreement No 730900 (H2020 INFRAIA-01-2016-2017 Action), (2) Contract "PREPOSE - PRE-and POst Seismic Events analysis" (Contract n.2500033423/2018 ENI Spa - University of Naples Federico II), and (3) Project FLUIDS - Detection and tracking of crustal fluid by multiparametric methodologies and technologies (PRIN 2017 - Prot. 20174X3P29). undergo attenuation, multi-path and site effects which pollute the recorded signals. In addition, depending on the earthquake size, the heterogeneity of the rupture initiation, development, and arrest, can make the recorded signals complex, with irregularities and details which are difficult to reproduce with standard source and wave propagation models.

The fault average seismic moment (moment magnitude), the rupture length (area) and the derived (static/dynamic) stress release are the principal macro-scale parameters which characterize the earthquake rupture strength and dynamics and provide relevant information on their scaling behavior with the event magnitude and on the earthquake impact in terms of predicted/observed strong ground shaking.

The estimation of earthquake source parameters from records of a dense, near-source seismic network is usually performed using signal processing techniques applied in frequency domain as a rather standard practice in seismological laboratories operating local and regional networks. Grossly, two basic modeling approaches are used. The first, non-parametric method, determines the magnitude and the other relevant source parameters through a multi-variate regression of empirical attenuation relations, with the signals filtered in consecutive and progressively increasing frequency bands (e.g., [1], [2]). The second, parametric method, determines the seismic moment, source radius/length and static stress drop from a spectral fitting procedure applied to displacement spectra, based on the predicted body-wave radiation of circular/rectangular fault ruptures (e.g., [3]-[6]). Indeed, since the pioneering work of Brune [7] several dynamic and kinematic rupture models have been proposed assuming a circular/rectangular rupture, to relate the observed parameters of the far-field displacement spectrum (low-frequency plateau and corner frequency) to the seismic moment $\left(M_{o}\right)$ and rupture radius $(a)$ /length $(L)$ [3], [8]-[11]. The critical issues related

E to the use of spectral techniques are the choice of the time window to be selected for the analysis which necessarily includes the pre-event noise and the secondary multi-path arrivals other that the direct waves and the appropriate correction or modelling of the frequency dependent anelastic attenuation and site amplification effects.

The authors would like also to express their appreciation to Gaetano Festa (Naples Federico II, Italy) for his constructive comments on the original work leading to the preparation of this article. (Corresponding author: Aldo Zollo.) A. Zollo, S. Nazeri, and S. Colembelli are with Department of Physics "E. Pancini", University of Naples Federico II, Naples, Italy (aldo.zollo@unina.it; sahar.nazeri@unina.it; simona.colombelli@unina.it). 
On the other hand, the modelling of the body-wave signal in time-domain as a straightforward approach to determine the earthquake source parameters is less diffused, except for the cases in which high-quality first $\mathrm{P}$ - and $\mathrm{S}$-wave signals are available [3], [12]. The apparent Source Time Function (STF) of earthquakes is indeed related and directly retrievable from the recorded $\mathrm{P}$ and $\mathrm{S}$ displacement waveforms at a set of well distributed stations around the epicenter. However, the timedomain methods are limited by the difficulty to retrieve noisefree, displacement waveforms from short-period and acceleration time-series.

In this paper we propose an original time-domain, P-wave based, parametric method to determine the earthquake seismic moment, rupture size and stress-drop. The method originates from recent developments in real-time signal processing for earthquake early warning, aimed at obtaining a rapid assessment of the source magnitude and potential shaking impact [13]-[16]. In the previous studies, the time evolution of the logarithm of the peak displacement amplitude of high-pass filtered P-wave signals (named LPDT curve), is used as a proxy for the apparent Source Time Function (STF). The modeling of the LPDT curve allows estimating the peak amplitude of the STF and the rupture half-duration. Indeed, when averaged over several well distributed near-source sensors, the resulting LPDT curve shows a characteristic exponential-rise behavior, with a nearly monotonic increase up to a corner time and a plateau level, both scaling with the earthquake magnitude. While the distance-corrected, average plateau level is a proxy for the seismic moment, the corner time of the LPDT curves is used to estimate the source radius of an expanding rupture assuming the circular and kinematic model of Sato \& Hirasawa [8] (hereinafter S\&H73) [14], [15]. The S\&H73 model assumes a uniform rupture velocity and a decreasing slip rise-time from the center to the border of the fault and predicts a neartriangular $\mathrm{P}$ and $\mathrm{S}$ far-field waveform whose amplitude and duration are controlled by the rupture directivity. The S\&H73 model is constructed to satisfy the Eshelby [17] solution of a static crack, at each instant during the rupture evolution and thus depends on unknown parameters, such as seismic moment, rupture velocity and static stress-drop.

Here we adopted a generalized source model, where the farfield P-waveform radiated by a point- source is approximated by an isosceles triangular source time function [18], whose parameters (peak amplitude and half-duration) are estimated from the modeling of LPDT curves through the measurement of their plateau and corner-time. In the proposed approach, the parameter estimation does not depend on the kinematic model used to infer the source parameters. This approach is conceptually like the standard displacement spectral fitting for the source parameter determination, where the spectral shape is generally described by a low-pass, response filter curve having the asymptotic low frequency spectral level, the corner frequency and the high frequency decay as characterizing parameters.This new modelling procedure is a rapid, timedomain parametric technique to estimate the seismic moment, the rupture radius (length) and static stress drop from the joint measurement of plateau level and corner time of the curves.

\section{METHODS}

A. Far-Field P-wave displacement from a point-source, double couple source, triangular moment rate function

In a homogeneous, elastic, half-space Earth model, the farfield $\mathrm{P}$-wave displacement radiated from a point-source rupture [19] is described by:

$\mathbf{u}^{P}(\mathrm{t})=\frac{\mathrm{F}_{\mathrm{S}} \mathrm{R}_{\vartheta \varphi}}{4 \pi \rho \mathrm{V}_{\mathrm{p}}{ }^{3}} \frac{1}{R} \dot{\mathrm{M}}_{\mathrm{o}}(t) \widehat{\boldsymbol{u}}_{P}$

where $\mathrm{R}_{\vartheta \varphi}$ is $\mathrm{P}$-wave radiation pattern coefficient, $\mathrm{F}_{\mathrm{s}}$ is free surface factor, $\rho$ is density, $V_{p}$ is P-wave velocity in the given area, $\mathrm{R}$ is hypocentral distance and $\dot{\mathrm{M}}_{\mathrm{o}}(t)$ is the moment rate function (whose time integral is the seismic moment $M_{o}$ ). $\widehat{\boldsymbol{u}}_{P}$ is the P-wave polarization direction, which we assume here as the vertical direction, so that only the vertical component of the ground motion is considered for the analysis. By integrating along the time both members, eq. (1) becomes:

$\Omega_{o}=\int \mathbf{u}^{P}(\mathrm{t}) d t=A^{\prime} \frac{M_{o}}{R}$

where $\Omega_{o}$ is the area beneath the P-wave displacement pulse, the integral is extended over its time width and $A^{\prime}=\frac{\mathrm{F}_{\mathrm{S}} \mathrm{R}_{\vartheta \varphi}}{4 \pi \rho \mathrm{V}_{\mathrm{p}}{ }^{3}}$ is a constant factor, depending on the medium. Assuming that the moment rate function of the earthquake is an isosceles triangular function, with parameters $P_{D}$ (peak amplitude of the triangle) and $T_{D}$ (duration of the triangle), the area can be obtained as:

$\Omega_{o}=\frac{P_{D} T_{D}}{2}=A^{\prime} \frac{M_{O}}{R}$

from which we obtain the P-peak displacement as a function of the seismic moment and source duration:

$P_{D}=2 A^{\prime} \frac{M_{O}}{R T_{D}}$

In the hypothesis of a circular fault with uniform rupture velocity $\mathrm{V}_{\mathrm{R}}$ and final radius $a$, the pulse duration $\mathrm{T}_{\mathrm{D}}$ at $\mathrm{a}$ receiver located at a fault view angle $\theta(\theta$ is the angle between the normal at the fault plane and the $\mathrm{P}$-wave ray at the rupture nucleation point) is derived by geometric calculations using the Fraunnhofer approximation [18]:

$\frac{T_{D}}{2}=\frac{a}{V_{R}}\left(1-\frac{V_{R}}{V_{P}} \sin \theta\right)$

and its average value over the angle $\theta$ is:

$\frac{\bar{T}_{D}}{2}=a\left(\frac{1}{V_{R}}-\frac{2}{\pi V_{P}}\right)$

The source radius of a circular rupture is related to the seismic moment $M_{o}$ and static stress-drop $\Delta \sigma$ through the Keilis-Borok [19] formula: 
$a=0.75915\left(\frac{M_{o}}{\Delta \sigma}\right)^{\frac{1}{3}}$

by replacing equations (6) and (7) in equation (4) we obtain:

$P_{D}=2 \frac{A^{\prime}}{K} \frac{M_{O}{ }^{\frac{2}{3}}}{R}$

where the constant $K=1.5183\left(\frac{1}{V_{R}}-\frac{2}{\pi V_{P}}\right) \Delta \sigma^{-1 / 3}$ depends on the P-wave velocity, rupture speed and stress-drop. Taking the base-10 logarithm, equation 8 reproduces the standard attenuation relation for the P-peak displacement:

$\log P_{D}=A+B \log M_{o}+C \log R$

with $A=\log \frac{2 A \prime}{K}, B=\frac{2}{3}$ and $C=-1$, where $\mathrm{C}$ is the geometrical attenuation factor for a homogeneous and elastic Earth velocity model. Eq.9 can be used to estimate the seismic moment of an earthquake from the measurement of the P-peak displacement, assuming known values of the rupture velocity and static stress-drop.

\section{B. A NEW METHOD FOR BUILDING THE LPDT CURVE}

The original method to construct the LPDT curve required three main steps (see figure 1) : (1) the measurement of the logarithm of the maximum $\mathrm{P}$-amplitude at all available stations within the same time window from the P-onset, (2) the correction of the amplitude for the distance attenuation (green curve in Figure 1a for an individual station) and (3) the average of all distance-corrected peak displacement values (green curve in Figure 1b for a given event) [13]-[15], [20].

In this work, the proposed new method computes the final curve as the time-varying, maximum amplitude of the distancecorrected averaged peak displacement values (red curve in Figure $1 \mathrm{~b}$ for a given event) according to the formula:

$\operatorname{LPDT}(t)=\max \left\{\left\langle\log P_{D}^{C}\right\rangle(t)\right\}_{t_{0}}^{t}$

where,

$\log P_{D}^{C}(t)=\log P_{D}(t)+\log R$

and $t_{o}$ is the first P-arrival time. In the new approach, the log and max operators are inverted with respect to the original method of Nazeri et al. [15]. Indeed, the first part of this new algorithm is like the basic assumption of the B- $\Delta$ approach implemented in Japan earthquake early warning system to estimate the epicentral distance and magnitude from initial slope of the acceleration waveform in a logarithm scale [21][23]. Especially in the case of noisy P-records, with multi-path arrivals and non-monotonic increase of the initial displacement waveforms, the new method better reproduces the expected shape of the far-field apparent (e.g., averaged over many stations) waveform up to the corner time and the plateau (Figure $1 b)$. Figure $1 \mathrm{~b}$ shows an example of the LPDT curve of a given event following the previous and new algorithms explained above, shown with the green and red curves, respectively.

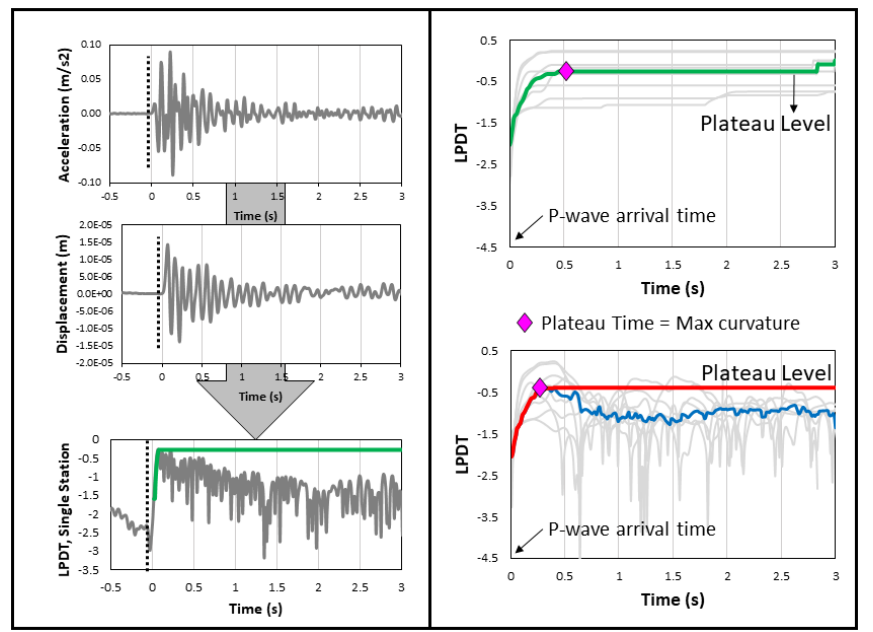

(a)

(b)

Figure 1. a. Different steps starting from the origin acceleration waveform (top panel) to bottom panel showing: the absolute displacement amplitude (grey curve); the absolute peak displacement amplitude (green curve) corrected by hypocentral distance in logarithmic scale. The high-passed filtered displacement signal shown in the middle panel is obtained after two times integration of the acceleration signal. The black dotted line shows the P-wave onset. b. The green and red curves are the LPXT curve of a given event following the previous and new algorithms explained in the text, respectively. The blue curve is the average (at least 5 stations are required) of absolute displacement normalized to distance. The magenta diamond refers to the plateau corner time.

Figure 2 shows two examples of the new generated LPDT curves for two large events in the analysed dataset (see section Data, Analysis and Results). For any given earthquake, three LPDT curves are built, e.g., the average ( $L P D T_{\text {Average }}$, and $\pm 1-$ sigma curves $\left(L P D T_{ \pm \mathrm{SD}}\right.$. While the curve parameter estimates (plateau level and corner time) are inferred from the curve $L P D T_{\text {Average }}$ their uncertainties are evaluated from the the \pm 1 - sigma curve and are finally used to obtain the errors on source parameters (seismic moment, source radius and stress drop).

C. Fitting of the LPDT and estimate of the plateau level and corner time

To estimate the plateau and corner time of the LPDT curves, we model the curves with a 3-parameter, exponential function [20], [24]:

$\operatorname{LPDT}(t)=y_{o}+P_{L}\left(1-0.5\left(e^{-\frac{t}{T_{1}}}+e^{-\frac{t}{T_{2}}}\right)\right)$

where $P_{L}, T_{1}$ and $T_{2}$ are curve-fitting parameters estimated by a non-linear best-fitting procedure. Here the parameter $y_{o}$ is constrained to the intercept of the curve. The plateau level is then obtained as $P_{L}{ }^{*}=y_{o}+P_{L}$. As for the corner time of the curves we developed a specific algorithm to evaluate the timevarying curvature of the fit-curve [25] and measure of time at which it approaches to zero to begin the plateau level (Figure $1 b)$. By approximating the measured corner time $\left(T_{c}\right)$, as the half-duration of a triangular STF (Figure 2c, Nazeri et al., [15]) $\left(T_{c} \approx \frac{\bar{T}_{D}}{2}\right)$, eq.6 can be used to obtain an independent estimation of the source radius $a$, assuming that the rupture velocity and 
P-wave velocity are known.

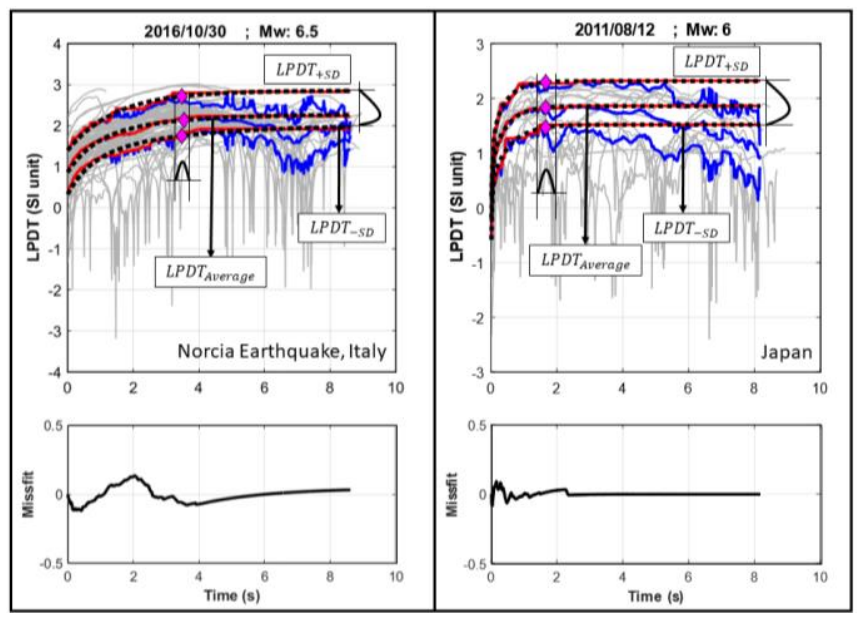

Figure 2. Two examples of the generated LPDT curves for two large events, in both panels, the grey curves are the logarithm of the P-amplitude measured at different stations and corrected for the distance attenuation. Blue curves are the average ( $\mathrm{LPDT}_{\text {Average }}$, plotted at the middle) and plus/minus one standard deviation $\left(\mathrm{LPDT}_{ \pm \mathrm{SD}}\right)$ of the scatter at each time step. The dotted black curves show the best fit to the red curves representing the maximum amplitude in the expanded time window. $\mathrm{T}_{c}$, the corner time of the plateau level is shown with pink diamond. The bottom panels show the mis fit, residual (LPDT ${ }_{\text {fit }}-\mathrm{LPDT}_{\mathrm{obs}}$ ) LPXT curves of all selected earthquakes for both regions, Japan (top) and Italy (Italy).

Moreover, by merging both equations 6 and 7, and Hanks \& Kanamori (1979) formula $\left(\log \left(M_{0}\right)=1.5 * M_{w}+9.1\right)$, the theoretical relation between $\log \left(T_{c}\right)$ and $\log \left(M_{0}\right)$ (and $M_{w}$ ) is expressed as $\log T_{C} \propto\left(\frac{1}{3}\right) \log \left(M_{0}\right) \propto 0.5 M_{w}$, while from eq. 9 , the plateau level is theoretically related to $\log \left(M_{0}\right) / M_{w}$ as $\log P_{L}^{*} \propto\left(\frac{2}{3}\right) \log \left(M_{0}\right) \propto M_{w}$.

D. Estimation of the source parameters: Seismic moment, rupture radius and stress-drop

The LPDT fitting procedure described in 2.3 provides in output the corner time $T_{c}$ in addition to the plateau level $P_{L}^{*}$. These two parameters can be jointly used to obtain an independent estimation of the seismic moment, source radius and stress-drop. For source parameter determination of smallto-moderate size earthquakes, the rupture velocity is generally assumed from the kinematic rupture models that are adopted to evaluate the rupture size. For circular rupture models, a typical value of $V_{R}=0.9 V_{S}$ is used where $V_{S}$ is the shear wave velocity [7]-[9]. In the hypothesis of the corner time as a proxy of the half-duration of the triangular source time function, $T_{c}=\frac{\bar{T}_{D}}{2}$, eq. 4 can be approximated as:

$P_{D}^{*} \cong A^{\prime} \frac{M_{O}}{R T_{C}}$

and the seismic moment

$\log M_{o}=\log P_{D}{ }^{*}+\log \frac{T_{C}}{A^{\prime}}+\log R \cong P_{L}^{*}+\log \frac{T_{C}}{A^{\prime}}$

Finally, eq.6, 7 and 14 are used to estimate the seismic moment $M_{o}$, the source radius $a$ and stress drop $\Delta \sigma$, respectively.

\section{E. Source parameter uncertainty estimation}

Using the error estimation of both $T_{c}$ and $P_{L}{ }^{*}$ values from the observed LPDT curves (Figure 2) as $\Delta T_{C}$ and $\Delta P_{L}{ }^{*}$, we illustrate here how the uncertainty on three source parameters i.e., seismic moment, rupture radius and stress-drop is inferred.

Based on eq. 14, the error estimation of $\log M_{0}$ is obtained as:

$\Delta\left(\log M_{o}\right)=\Delta P_{L}^{*}+\frac{\Delta T_{C}}{T_{C}} \log _{10} e$

where $\Delta M_{0}$ can be derived as:

$\Delta M_{0}=\frac{M_{O} \Delta\left(\log M_{O}\right)}{\log _{10} e}$

As for the error estimation of the source radius, using the eq. 6 , we obtain:

$\Delta a=\frac{\Delta T_{C}}{\left(\frac{1}{V_{R}}-\frac{2}{\pi V_{P}}\right)}$

Fina1lly, using the eq. 7, the error estimation of the stress drop is computed as:

$\Delta \sigma=\sigma\left(\frac{\Delta M_{0}}{M_{0}}-3 \frac{\Delta a}{a}\right)$

\section{$F$. Correction for the anelastic attenuation effect ( $Q$-filter)}

Eq. 1 represents the far-field displacement in an elastic, homogeneous Earth propagation medium and it does not account for anelastic attenuation effects which can modify the amplitude and frequency content of the recorded displacement waveform. Following the standard theory [26], the anelastic attenuation function is parametrized by the Q-parameter, which expresses the ratio between the amount of radiated energy dissipated in rock materials for frictional (inelastic) phenomena during the wave propagation. The primary effect of attenuation on seismic signals is the broadening and amplitude decrease of the source pulse, which becomes relatively important as a function of the $\mathrm{Q}$ value, the recording distance, and the frequency content of the radiated signal. In general, significant pulse distortions are expected in conditions of low Q (less than few tens), large recording distances (greater than 50-100 km), and high frequencies (greater than $10-20 \mathrm{~Hz}$ ).

The basic parameter that characterizes the seismic attenuation function is the Q-normalized travel-time " $\mathrm{t}$-star", $t^{*}=\frac{T_{P}}{Q_{P}}\left(T_{P}\right.$ is the P-wave travel-time and $Q_{P}$ is the quality factor), where $\mathrm{Q}$ is assumed constant with frequency. This is a reasonable and commonly used approximation for applications such as the source parameter estimation from body wave time and frequency domain signals [27]. A possible strategy for the correction of $\mathrm{P}$-wave signals for the $\mathrm{Q}$ anelastic attenuation effect would require to deconvolve the recorded signal by the attenuation operator (i.e., whose analytical, approximated expression for a linear attenuation model is given in eq.36 of 
Kjartansson, [26]) at any station before proceeding on the computation of the LPDT curves as described in section 2.2. This implies the prior knowledge of the $Q_{P}$ quality factor for the investigated area and the travel time of the first P-wave as determined for each recording stations from the earthquake location and Earth P-velocity model.

The Q-pulse deconvolution is a critical issue in time-domain signal processing operations, possibly producing artefacts due to the presence of undesired high noise amplitudes in the considered frequency range of earthquake signals.

We propose here an alternative approach, that uses instead the less contaminant convolution operation as the basic signal processing [28]. In the hypothesis of a triangular apparent moment rate function (MRF), the parameters corner time $\left(T_{c}\right)$ and plateau level $\left(P_{D}{ }^{*}\right)$, measured from the LPDT curves, define the half-duration and peak amplitude of the observed logarithmic apparent displacement waveform (ADW), which is related to the MRF. Let us recall that the ADW is obtained by averaging the logarithmic displacement waveforms recorded at several stations located at different distances from the earthquake epicenter, each of them possibly affected by the travel path anelastic attenuation effect. Therefore, the observed ADW can be considered as the summation of anelastic attenuated pulses recorded at each station and resulting from the convolution of the source triangular function with the attenuation function, with the station parameter $t^{*}$.

To determine the half-duration and peak amplitude parameters of the Q-corrected triangular source function, we implemented a global search strategy based on the recursive simulation of anelastic attenuated triangles at the set of recording stations for a given triangular source parameter couple $\left(T_{c}\right.$ and $\left.P_{D}^{*}\right)$ with a known value of the quality factor $Q_{P}$, computation of the synthetic LPDT curve, measure of the related $T_{c}$ and $P_{D}^{*}$ parameters and best-fit matching with the observed values. For a given earthquake, the synthetic Qattenuated triangles are generated at each station using the CREWES software for Q modelling and analysis [29] that numerically implements the linear attenuation model of Kjartannson [26]. To fasten the optimization strategy, a precomputed table of synthetic $T_{c}$ and $P_{D}^{*}$ parameters is built by the regular sampling of a discretized triangle half-duration, peak amplitude space. The limits of the explored range of parameters are set based on the interval of triangle parameters associated with a wide range of seismic moment $\left(\log \left(M_{0}\right)=\right.$ $\log \left(M_{0_{L P D T}}\right) \pm 2 * \Delta\left(\log M_{0_{L P D T}}\right)$, ) ) and stress drop values $\left(\Delta \sigma^{\min }=0.01 \mathrm{MPa} ; \Delta \sigma^{\max }=100 \mathrm{MPa}\right)$, to which the triangle parameters are linked through eq. 6,7 and 8 . The best fit source triangle parameter values are chosen as the one that minimizes the sum of the Chi-squared variables of $T_{c}$ and $P_{D}^{*}$ obtained by combining synthetic and observed values (e.g. $\left.\mathrm{X}^{2}=\frac{\left(T_{C}^{O B S_{-}} T_{C}^{S Y N}\right)^{2}}{T_{c}^{S Y N}}\right)$. Finally, the found best-fit values of $T_{c}$ and $P_{D}^{*}$ are used to estimate the seismic moment, source radius and stress-drop through eq.6,7 and 14 .

\section{G. First-order correction for site amplification/attenuation} effect

In our approach, eq.9 is used to evaluate the azimuthally averaged P-peak displacement amplitude in the hypothesis that for a single event, possible site P-wave amplification/attenuation effects are averaged-out among different stations and local site conditions. However, when waveform data are available for many past earthquakes recorded at the same site, eq. 9 can be generalized including a site term $S_{j}$ :

$\log P_{D}^{i j}=A+B \log M_{o i j}+C \log R_{i j}+S_{j}$

where $\mathrm{i}$ and $\mathrm{j}$ are the indexes of the earthquake and station, respectively. Extracting the P-peak log-displacement values at the same station for different events, the term $S_{j}$ can be estimated as the average or median value of the difference:

$S_{j}=\log P_{D o b s}^{i j}-\left\langle\log P_{\text {Dtheo }}^{i j}\right\rangle_{i}$

with $\log P_{D o b s}^{i j}$ is the observed log-displacement for a given couple i, j and $\left\langle\log P_{D \text { theo }}^{i j}\right\rangle_{i}$ is the event-averaged logdisplacement computed from eq. 9 which does not include site term. In this study we did not apply the site amplification correction and assumed $S_{j}=0$.

\section{DATA ANALYSIS AND RESULTS}

In order to implement and test the proposed technique, we choose a dataset of events with moment magnitude ranging from $M_{W} 2.5$ to $M_{W} 6.5$ as extracted from the available databases of earthquakes occurred in Central Italy (belongs to the 2016-2017 Central Italy seismic sequence; 135 events) and Japan (occurred between 2007-10-06 and 2019-07-22; 83 events) provided by INGV (Istituto Nazionale di Geofisica e Vulcanologia) and JMA (Japan Meteorological Agency), respectively (Figure 3). 

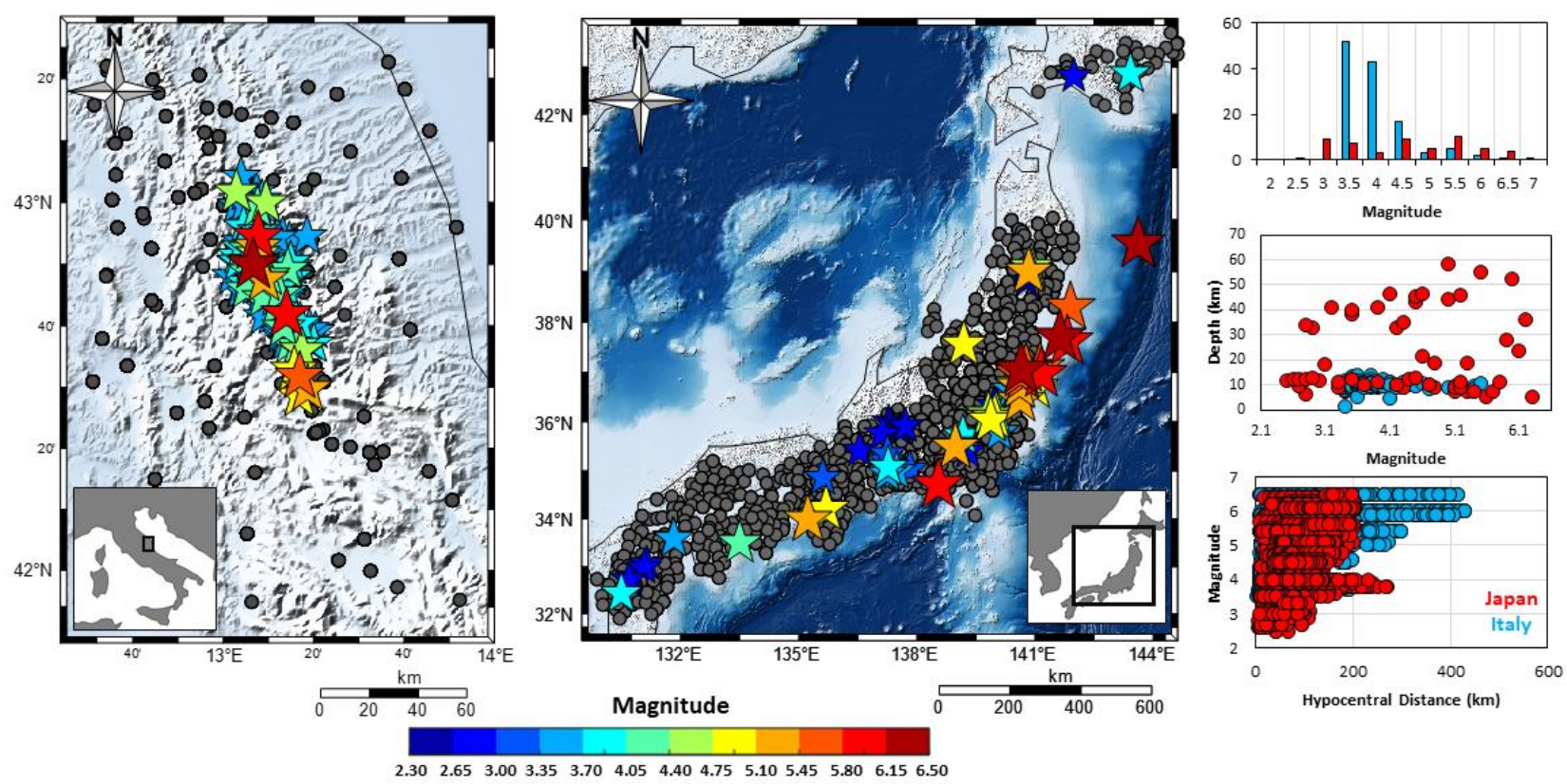

Figure 3. The maps show the distribution of all selected events (coloured stars) with a variable size, depending on the magnitude and position of the stations used for the analysis (dark grey circles). Distribution of magnitude, depth of the events as a function of magnitude, and the number of records as a function of hypocentral distance are shown in the bottom row.

After a preliminary evaluation of the data quality, the original dataset is reduced to 124 events for Central Italy and 53 events for Japan, covering the same magnitude range. About $66 \%$ of the Japanese events used in this study (53 earthquakes) are crustal (hypocentral depth $\leq 30 \mathrm{~km}$ ), whereas $34 \%$ are subcrustal (hypocentral depth $>30 \mathrm{~km}$ ), [30], [31]. Moreover, the magnitude reported by JMA $\left(M_{J M A}\right)$ for the Japanese dataset is converted to $\mathrm{Mw}$ using the scaling relation of $M_{W}=1.037$ $( \pm 0.008) M_{J M A}-0.297( \pm 0.034)$, obtained from a linear orthogonal regression on data of about 1,826 earthquakes $\left(M_{J M A} 2.7-8\right)$ throughout Japan [31] with a rms residual of 0.22 and 0.24 for whole data and for small events $\left(M_{J M A} \approx 2.7-3.5\right)$ respectively. The $\mathrm{P}$-wave arrival time has been manually picked on a database of 2596 (Italy, 125 events) and 1159 (Japan, 54 events) vertical acceleration waveforms recorded at an hypocentral distance smaller than $100 \mathrm{~km}$.

During the data processing step, a $0.075 \mathrm{~Hz}$ high-pass Butterworth filter is applied to the displacement records to remove possible baseline effects arising from the lowfrequency noise amplification and twice integration of the accelerometric records.

For each event, the LPDT curves have been built using the procedure described in 2.2, and the parameters $\boldsymbol{P}_{\boldsymbol{L}}{ }^{*}$ and $\boldsymbol{T}_{\boldsymbol{c}}$ are therefore estimated through the LPDT modeling step with the corresponding uncertainties shown with error-bar in Figure 4. Figure 4a shows the observed LPDT curves for both studied areas Central Italy and Japan, and Figure $4 \mathrm{~b}$ and $4 \mathrm{c}$ present the two corresponding parameters of the LPDT curves i.e., the plateau level (panel b) and the corner time (panel c) before and after correcting by the Q-filter method.

In this study we applied the correction for anelastic attenuation assuming a constant- $Q$ attenuation model and the estimation of the average crustal P-wave quality factor in the two seismic regions, Central Italy, and Japan, as available from the literature. In the Supplemental Material we illustrate how the choice of density $\rho, V_{P}$-to- $V_{S}$ ratio and $Q_{P}$ can significantly affect the stress-drop estimates, in the proposed or other techniques, the reason why the specific used values and models for attenuation and velocity should be considered when comparing the stress-drop estimates in the same or different seismic regions. The values of average $\rho, V_{P}$-to- $V_{S}$ ratio and $Q_{P}$ used in our case studies of Central Italy and Japan are summarized in Tables $1 \mathrm{~S}$ and $2 \mathrm{~S}$.

As for the attenuation parameter for Central Italy we refer to the study of Chiarabba et al. [32] who performed a velocity and attenuation tomographic study of the shallow crustal volume in the region of the 1997 Umbria Marche earthquake sequence, occurred several tens of $\mathrm{km} \mathrm{NW}$ of the 2016-2017 Central Italy sequence analyzed in this paper. Although their paper is mostly focused on retrieving and interpreting the spatial variability of the $V_{P}$-to- $V_{S}$ and $Q_{P}$-to- $Q_{S}$ ratios as an indicator of the presence of fluid-saturated rocks and fluid migration, the inferred $Q_{P}$ shows a marked increase from a $Q_{P}<100$ to $Q_{P}>200$ at depth of about $3 \mathrm{~km}$, with an average value of $Q_{P}=150$ (the average value used in our study) within the upper $8-10 \mathrm{~km}$ crustal layer. A similar range of $Q_{P}\left(Q_{P}=50-200\right)$ estimates in Central Italy has been found by De Lorenzo et al. [33]. We investigated the variability of the source parameter estimates with different $Q_{P}$ values in the range 80-300, thus providing an interval of inferred average stress-drop between $\langle\Delta \sigma\rangle=0.29(0.24-$ $0.34)$ and $\langle\Delta \sigma\rangle=0.61(0.49-0.76) \mathrm{MPa}$.

For Japan, we refer to the updated attenuation tomography study of Wang and Zhao [34] who derived the P ad S attenuation tomography models using $300 \mathrm{k}+\mathrm{P}$-wave $\mathrm{t}$-star data measured from spectra of local earthquakes recorded by the dense HI-Net Japanese seismic network. This study shows a $Q_{P}$ increasing with depth, from $Q_{P}=299$ at $10 \mathrm{~km}$ depth to $Q_{P}=820$ at $60 \mathrm{~km}$ depth (we used these values in this study for crustal and subcrustal events respectively in our study. 
TABLE I

List of the known values of the medium and source parameters used in this study for both Central Italy and Japan (see Tables $1 \mathrm{~S}$ and 2S).

\begin{tabular}{|l|l|r|r|r|r|c|}
\hline \multicolumn{2}{|c|}{ Region } & $\begin{array}{c}V_{P} \\
(\mathrm{~km} / \mathrm{s})\end{array}$ & $V_{P} / V_{S}$ & $\begin{array}{c}\alpha^{\prime} \\
V_{R} / V_{S}\end{array}$ & $\mathrm{~F}_{\mathrm{S}} * \mathrm{R}_{\vartheta \varphi}$ & $\begin{array}{c}\rho \\
(\mathrm{kg} / \mathrm{m} 3)\end{array}$ \\
\hline \multicolumn{2}{|c|}{ Italy } & 6.00 & 1.9 & 0.9 & 1 & 2.7 \\
\hline \multirow{2}{*}{ Japan } & Crustal & 5.20 & 1.7 & 0.9 & 1 & 2.9 \\
\cline { 2 - 7 } & Subcrustal & 5.72 & 1.7 & 0.9 & 1 & 2.9 \\
\hline
\end{tabular}

The source parameter estimates obtained in this study and their scaling relationship with magnitude are represented in Figure 5. To this end, we use the known values of the medium and source parameters required in formulae (6), (7) and (12), as they are listed in Table 1 for both Central Italy and Japan.

We first compare in a log-log plot (Figure 5a), the LPDTderived seismic moment vs the same quantity as available from the Italian and Japanese catalogues. The two quantities scale following approximately the 1:1 linear trend, all along the whole explored magnitude range.

The rupture half-duration (Figure $4 \mathrm{c}$ ) and rupture radius (Figure 5b), as estimated from the corner time of LPDT curves, show a constant stress-drop scaling with magnitude, for the whole explored magnitude range, with a consistent distribution for Japanese and Italian earthquakes. The nearly constant stressdrop trend with magnitude is better evident in Figure $5 \mathrm{c}$, but with a slightly different frequency distribution (right side histograms) for Japan and Italy, and larger median values for Japanese $(\Delta \sigma=0.60(0.42 ; 0.87)$ and $1.53(1.01 ; 2.31) M P a$, with the $95 \%$ confidence limits given in parenthesis, for crustal and subcrustal events, respectively) than for Italian earthquakes $(\Delta \sigma=0.36 \mathrm{MPa}(0.30 ; 0.44))$.

\section{DISCUSSION}

We propose a new time-domain technique aimed at estimating the source parameters of micro-to-moderate earthquakes, based on the measurement of the logarithmic Pwave peak amplitude as a function of the time from the first arrival. This method uses the available information from Psignal amplitudes recorded at a local seismic network as the Pwavefront propagates along the array and it is therefore feasible to be incorporated in a near-real-time, automatic procedure for earthquake source parameter determination. The method adopts a parametric modelling approach by assuming the moment rate function of the earthquake as represented by an isosceles triangular displacement waveform, whose peak amplitude and half-duration are theoretically related to the seismic moment, source radius and static stress release.

To implement this method, we assume that the earthquake location, the first P-arrival time pickings at the network seismic records and the velocity and attenuation model parameters are preliminary known. While here we used a formulation based on a uniform velocity model, this can be easily generalized to the case of a vertically heterogeneous medium using the appropriate values for receiver/source velocity, density, and geometrical spreading [5].

The correction for the geometric and anelastic attenuation effects is a critical issue for the reliable and accurate estimation of source parameters both in time and frequency domain, especially considering small magnitude events recorded at large distances. Here we propose the application of a Q-filter algorithm, which requires the a priori knowledge of the average P-wave quality factor for the investigated propagation volume and allows to retrieve the unperturbed average triangular MRF, whose peak amplitude and half-duration are used to estimate the seismic moment and rupture radius.

We tested and validated the methodology by its application to two high quality earthquake waveform data sets recorded by accelerometric local networks in Central Italy and Japan, and covering a wide magnitude, distance, and depth range.

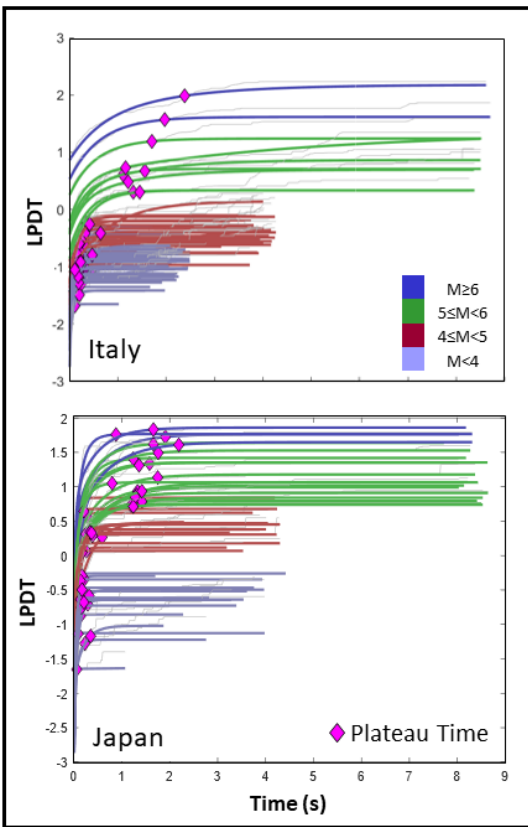

(a)

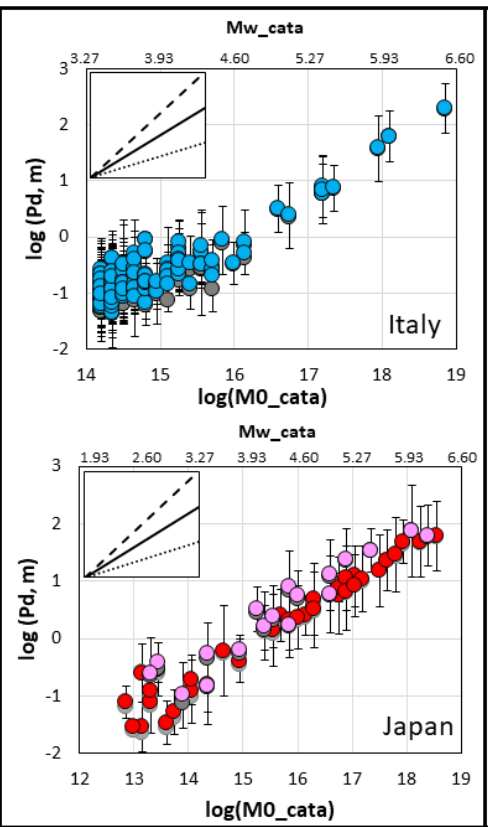

(b)

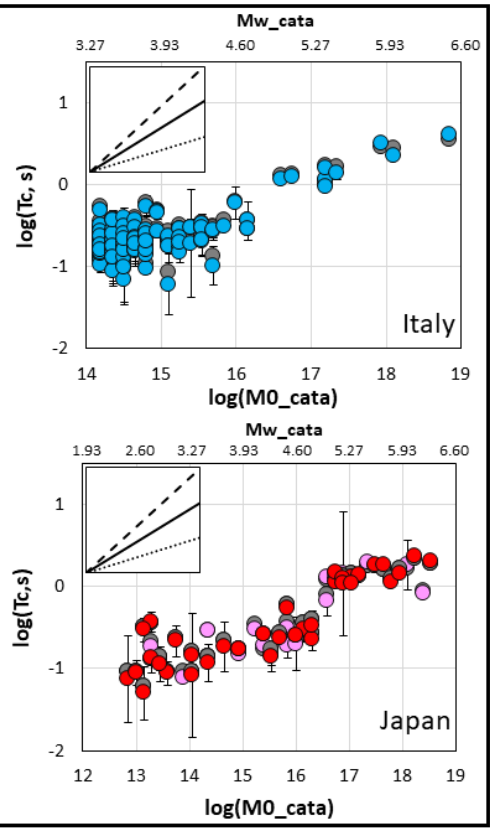

(c)

Figure 4. a. LPXT curves of all selected earthquakes for both regions, Italy (top) and Japan (bottom). b/c. peak amplitude (plateau level)/half duration (corner time of LPDT) of the STF as a function of the seismic moment reported by agencies before (gray circles) and after (top: blue circles for Italy, and bottom: red/pink circles for Japan, crustal and subcrustal respectively) applying the Q-filter in the logarithm scale. The small plot shows the 1:1 (black solid line), 1:0.5 (black dotted line), and 1:1.5 (black dashed line) relations. 


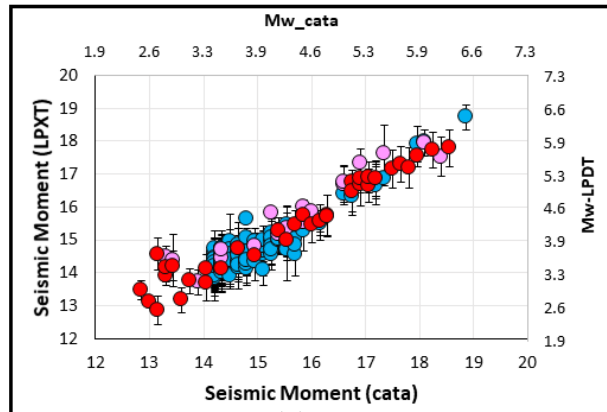

(a)

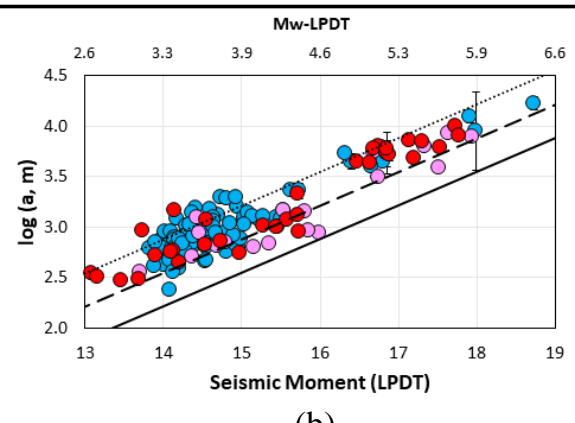

(b)

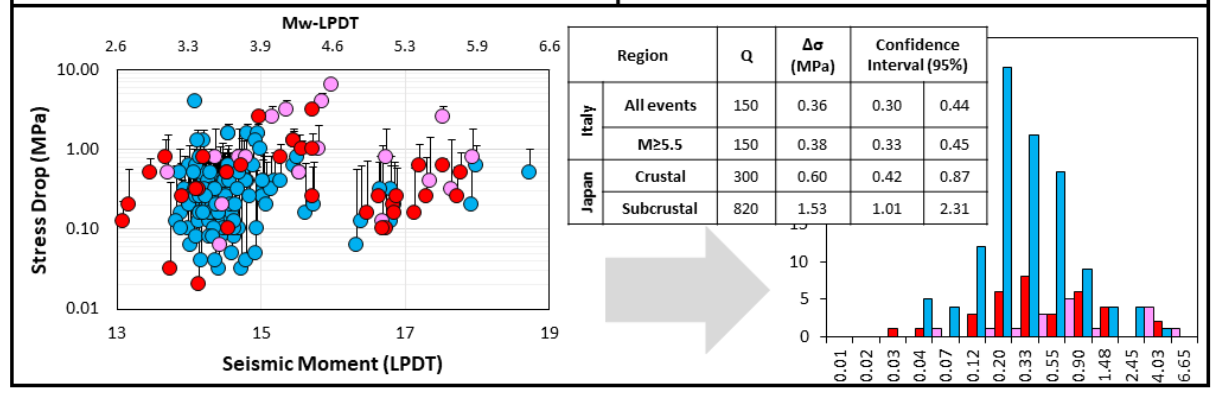

(c)

Figure 5. a. The estimated seismic moment using the LPDT method as a function of the seismic moment reported by agencies. b. Scaling of the source radius in terms of the estimated seismic moment using the LPDT method. The lines represent the theoretical scaling using the Keilis-Borok (1959) formula and assuming the constant static stress-drop values: 0.1 (dotted line), 1 (dashed line), and 10MPa (solid line). c. The estimated stress-drop for the individual earthquakes in the selected data sets. In all plots, the blue circles refer to Italy, and the red and pink circles represent the crustal and subcrustal events of Japan, respectively. The grey squares represent the values computed by Madariaga's (1977) formula, using the average rupture length as obtained by two different fault models (Cheloni et al. 2017; Xu et al. 2017).

In addition, we investigate the methodology performance when applied to earthquake records generated in two different tectonic settlements, as the thrust/strike-slip regime related to the subduction in Japan and extensional, normal faulting regime in Central Italy.

The retrieved LPDT curves for both regions show the characteristic smooth, ramp-like shape [13] with a sharp increasing amplitude vs time and a plateau level which increases with the size of the event. The rise time of the LPDT curves also increases with the magnitude, supporting the evidence of a corner-time which scales with the duration of the apparent source time function.

After correction for anelastic attenuation, the peak displacement and triangle source duration retrieved for the Central Italy and Japanese earthquake sequences analyzed in this study, show a scaling with moment magnitude that is consistent with the expected trends from theory (see section 2.3) $\left(\log P_{D} \propto M_{w}\right.$ and $\left.\log T_{C} \propto 0.5 M_{w}\right)$ in the hypothesis of a self-similar, constant stress-drop scaling (Figure $4 \mathrm{~b}$ and $4 \mathrm{c}$ ).

The methodology provides reliable estimates of the seismic moment and source radius of earthquakes down to $\mathrm{M}$ about 2.5. At smaller magnitudes, the main limitation can be the difficulty to discriminate extremely short corner times $(<0.05 \mathrm{sec})$, on low sampling rate records which prevents the accurate reconstruction of the ascending part of the LPDT curve up to the plateau. In principle, this difficulty can be overcome by using high-sampling velocimetric instruments, capable of providing good-quality integrated displacement waveforms.

In the explored magnitude range and for the two different seismic regions, the method retrieves source parameter estimates which confirm a constant stress-drop scaling of the rupture radius with seismic moment.
The found average stress-drop values and variability for the Japanese crustal earthquakes $(\Delta \sigma=0.60(0.42 ; 0.87) \mathrm{MPa})$ are well consistent with the static stress-drop distribution for the similar depth range as obtained by Oth et al. [30] and Oth [31] ( $\Delta \sigma=0.1-1 \mathrm{MPa})$, who derived the event source parameters by a non-parametric spectral inversion technique that accounts for both anelastic and geometrical attenuation and an omega-square source spectral model [7], [35]. The high spatial correlation between the crustal earthquake, low stress-drop values and heat flow variations led Oth [31] to propose a primary thermal control in the crustal earthquake generation process.

The average stress-drop of the 18 sub-crustal earthquakes estimated in our work $(\Delta \sigma=1.53(1.01 ; 2.31) \mathrm{MPa})$ is systematically higher than for crustal events, but our measured values are a factor 10 smaller than the ones obtained by Oth [31] $(\Delta \sigma=5-15 \mathrm{MPa})$.

A strong overestimation of the P-wave quality factor for the deepest Japanese events could originate an underestimation of the stress-drop with our proposed time-domain method. However, extremely low $Q_{P}$ values $(<100-200)$ that are not consistent with the recent attenuation tomography studies in Japan, would be required to justify stress-drop larger than 5 MPa.

However, like Oth [31] but not considering their absolute values, higher stress drops are observed for sub-crustal earthquake ruptures in Japan occurring along the subducting zone interface, but with an average value consistent with the subduction zone estimates $(\Delta \sigma=2.98 \pm 0.21 \mathrm{MPa})$ inferred from the global stress-drop variation study of Allman and Shearer [36]. The relatively higher stress-drop for sub-crustal earthquakes in Japan may be related to the strong coupling/ high 
seismic energy release of earthquakes occurring along the down-dip region [37].

As for the 2017 Central Italy earthquake sequence, the source parameters have been determined in several previous studies using non-parametric and parametric modelling approaches based on the inversion of the recorded S-wave spectra [6], [38], [39] and P-wave time-domain peak amplitude [15].

Our stress-drop estimates $(\Delta \sigma=0.36 \mathrm{MPa}(0.30 ; 0.44))$ are consistent with the mean value and $95 \%$ confidence limits derived by Bindi et al. [38] $(\Delta \sigma=0.57 \mathrm{MP}(0.08 ; 4.79 \mathrm{MPa})$,) using a non-parametric technique and a factor 4-5 smaller than estimates from Supino et al. [6] $(\Delta \sigma=2.1 \pm 0.3 \mathrm{MPa})$ using a non-linear parametric modelling of the S-displacement spectra.

In a similar moment magnitude range of our study, Wang et al. [39] used a two-step non-parametric generalized inversion technique applied to $\mathrm{S}$-wave displacement spectra. They showed the evidence for a step-like increase of stress-drop vs magnitude, with the smaller magnitude events $\left(M_{W}<5.0\right)$ characterized by an average stress-drop value $(\langle\Delta \sigma\rangle=$ $0.6 \mathrm{MPa}$, range: $0.1 \div 3 \mathrm{MPa}$ ), which is well consistent with our estimates, while the largest ones $\left(M_{W} \geq 5.0\right)$ have a significantly higher stress-drop value $(\langle\Delta \sigma\rangle=7.15 \mathrm{MPa}$, range: $5.5 \div 12.2 \mathrm{MPa}$ ), which led the authors to hypothesize a breakdown of the earthquake rupture self-similarity in the explored magnitude range for this specific seismic sequence.

In our study the retrieved rupture radius scaling with seismic moment (Fig. 5 b and c) seems to contradict this hypothesis, since a well constrained self-similar, constant stress-drop scaling is observed over about six orders of magnitudes of the seismic moment, with an almost similar stress drop released for the large and small magnitude events of the Central Italy sequence.

For both sub-crustal Japan and larger magnitude Central Italy earthquakes our stress-drop estimates are significantly smaller than the ones obtained by parametric and non-parametric spectral inversion techniques. This discrepancy might be related to the bias in spectral modelling techniques due to the possible presence of secondary, higher corner frequencies associated with the radiation of localized, high slip patches of the fault, which may lead to an underestimation of the total rupture size and then, higher stress drop values. In support of this hypothesis, the geodetic models of the 2016-2017 Central Italy earthquake sequence inferred from InSAR and GPS data [40], [41] show the evidence for the activation of localized high slip patches (slip $\sim 1 \mathrm{~m}$, radius $\sim 5 \mathrm{~km}$ ), during the rupture process of the larger magnitude events (August,8, $2016 \mathrm{Mw}$ 6.2; October, $10 \mathrm{Mw}$ 5.5; October,30 Mw 6.5; January, $18 \mathrm{Mw}$ 5.7). In this case, a factor 8 of stress-drop overestimation, would be produced by the measure of a predominant spectral corner frequency associated with high-slip fault areas with about the half radius of the whole ruptures area.

On the other hand, since the proposed LPDT time-domain method retrieves the rupture size and seismic moment from the half-duration and area of a station-averaged apparent moment rate function (assumed to have an isosceles triangular shape) it shows to be less sensitive to the complexity of the rupture development and $\mathrm{P}$-wave radiation processes, since the logdisplacement curve irregularities produced by localized fault patch radiation are smoothed-out by the apparent MRF average among stations located at different azimuth and distance from the radiating source.

\section{CONCLUSION}

In this article we illustrated the theory and application of a new method for earthquake source parameter determination (seismic moment, rupture radius and static stress drop) based on the modelling of $\mathrm{P}$-wave log-displacement amplitude versus time curves reconstructed from ground acceleration (or velocity) records acquired by a dense local seismic network.

The methodology grounds on a uniform speed, circular rupture model which assumes a triangular moment rate function, whose parameters, peak amplitude and duration, are related to the retrieved source parameters.

We further account for anelastic attenuation by applying a post processing Q-filtering technique, that requires the prior knowledge of the $\mathrm{P}$-wave quality factor for the investigated seismic volume. A first-order correction for site amplification/attenuation is proposed.

- The method has been applied in two different tectonic environments, Central Italy and Japan, to estimate the source parameters and rupture radius scaling relations of earthquakes spanning a relatively wide moment magnitude range. In both region we observe a self-similar, constant stress-drop scaling over the whole explored magnitude range. The differences in the average stress-drop values depend on regional stress and depth conditions.

- We found a significantly smaller average stress-drop for sub-crustal earthquakes in Japan and $M>5.5$ events in Central Italy relative to previous estimates using parametric and not-parametric spectral methods. The higher sensitivity of spectra to the seismic radiation from smaller but higher slip fault patches (e.g., higher corner frequency estimation) explains this discrepancy. Conversely, our proposed time domain technique is less sensitive to details of the rupture process, being grounded on azimuth and distance averaged log-displacement curves, which smooth-out irregularities produced by the rupture kinematic complexity.

The methodology is fast and accurate with the potential to be implemented in near-real-time, automatic procedures for rapid post-event assessment of the earthquake magnitude and size. We plan future improvements such as to integrate and combine $\mathrm{P}$ and $\mathrm{S}$ wave log-displacement curves, generalize the Earth propagation model to consider a vertical heterogeneous wave velocity, include the quality factor, $\boldsymbol{Q}_{\boldsymbol{P}}$, as an additional parameter to be determined along with the source and, finally, to extend the rupture model to consider great size earthquakes using a rectangular (Haskell-type) rupture model.

\section{ACKNOWLEDGMENT}

This work has been funded by several projects as: (1) Project "SERA - Seismology and Earthquake Engineering Research, Infrastructure Alliance for Europe" - Grant Agreement No 730900 (H2020 INFRAIA-01-2016-2017 Action), (2) Contract "PREPOSE - PRE-and Post Seismic Events analysis" (Contract n.2500033423/2018 ENI Spa - University of Naples Federico 
II), and (3) Project FLUIDS - Detection and tracking of crustal fluid by multi-parametric methodologies and technologies (PRIN 2017 - Prot. 20174X3P29).

\section{REFERENCES}

[1] Castro, R. R., Anderson, J. G., \& Singh, S. K. (1990). Site response, attenuation and source spectra of $S$ waves along the Guerrero, Mexico, subduction zone. Bull. Seismol. Soc. Am., 80:1481-1503.

[2] Picozzi, M., Bindi, D., Brondi, P., Di Giacomo, D., Parolai, S. \& Zollo, A., (2017). Rapid determination of P wavebased energy magnitude: in-sights on source parameter scaling of the 2016 central Italy earthquake sequence, Geophys. Res. Lett., 44, 4036-4045.

[3] Boatwright, J. (1980). A spectral theory for circular seismic sources; simple estimates of source dimension, dynamic stress drop, and radiated seismic energy. Bull. Seismological Soc. America 70 (1), 1-27.

[4] Abercrombie, R. E. (1995), Earthquake source scaling relationships from -1 to $5 \mathrm{ML}$ using seismograms recorded at $2.5 \mathrm{~km}$ depth, J. Geophys. Res., 100, 24,01524,036.

[5] Zollo, A., Orefice, A. \& Convertito, V., (2014). Source parameter scaling and radiation efficiency of microearthquakes along the Irpinia fault zone in southern Apennines, Italy, J. geophys. Res., 119, 3256-3275. doi:10.1002/2013JB010116.

[6] Supino, M., Festa, G., \& Zollo, A. (2018). A probabilistic method for the estimation of earthquake source parameters from spectral inversion: application to the 2016-2017 Central Italy seismic sequence. Geophysical Journal International, 218, 988-1007. https://doi.org/10.1093/gji/ggz206.

[7] Brune, J. N. (1970). Tectonic stress and the spectra of seismic shear waves from earthquakes. J. Geophys. Res. 75 (26), 4997-5009. doi:10.1029/JB075i026p04997.

[8] Sato, T., and Hirasawa, T. (1973). Body wave spectra from propagating shear cracks. J,Phys,Earth 21 (4), 415-431. doi:10.4294/jpe1952.21.415.

[9] Madariaga, R. (1976). Dynamics of an expanding circular fault. Bull. Seismological Soc. America 66 (3), 639-666.

[10] Madariaga, R., (1977). Implications of stress-drop models of earthquakes for the inversion of stress drop from seismic observations, Pure appl.Geophys., 115, 301-316.

[11] Savage, J. C. (1974), Relation between P- and S-wave corner frequencies in the seismic spectrum, Bull Seism. Soc. Am. 64, 1621-1627

[12] Stork, A.L. Verdon, J.P., \& Kendall, J.-M. (2014). The robustness of seismic moment and magnitudes estimated using spectral analysis Geophys. Prospect., 62, 862-878. https://doi.org/10.1111/1365-2478.12134.

[13] Colombelli, S., Zollo, A., Festa, G. \& Picozzi, M., (2014). Evidence for a difference in rupture initiation between small and large earthquakes, Nat. Commun., 5, 3958.

[14] Colombelli, S., \& Zollo, A. (2015). Fast determination of earthquake magnitude and fault extent from real-timePwave recordings. Geophys. J. Int. 202 (2), 1158-1163. doi:10.1093/gji/ggv217.

[15] Nazeri, S., Colombelli, S., \& Zollo, A. (2019). Fast and accurate determination of earthquake moment, rupture length and stress release for the 2016-2017 Central Italy seismic sequence. Geophys. J. Int. 217 (2), 1425-1432. doi:10.1093/gji/ggz097.

[16] Trugman, D., Page, M., Minson, S.E., \& Cochran, E. (2019). Peak ground displacement saturates exactly when expected: Implications for earthquake early warning. Journal of Geophysical Research, 124, 4642-4653. doi:10.1029/2018jb017093.

[17] Eshelby, J.D. (1957). The determination of the elastic field of an ellipsoidal inclusion, and related problems. Proceedings of the Royal Society of London. Series A. Mathematical and Physical Sciences, 241, 376 - 396.

[18] Duputel, Z., Tsai, V.V., Rivera, L. and H. Kanamori (2013) Using centroid time-delays to characterize source durations and identify earthquakes with unique characteristics, Earth and Planetary Science Letters, Volume 374, Pages 92-100, ISSN 0012-821X, https://doi.org/10.1016/j.epsl.2013.05.024.

[18] Aki, K., \& Richards, P. G. (2002). Quantitative seismology. Sausalito, CA: University Science Books.

[19] Keilis-Borok, V. (1959). On estimation of the displacement in an earthquake source and of source dimensions. Ann. Geofis. (Rome) 12, 205-214. doi:10.4401/ag-5718.

[20] Wang, Y., Colombelli, S., Zollo, A., Song, J., \& Li, S. (2021). Source Parameters of Moderate-To-Large Chinese Earthquakes from the Time Evolution of P-Wave Peak Displacement on Strong Motion Recordings. Frontiers in Earth Science. https://doi.org/10.3389/feart.2021.616229.

[21] Odaka, T., K. Ashiya, S. Tsukada, S. Sato, K. Ohtake, \& D.Nozaka (2003). A new method of quickly estimating epicentral distance and magnitude from a single seismic record, Bull. Seismol. Soc. Am. 93, 526-532.

[22] Yamamoto, S., S. Noda, \& M. Korenaga (2012). An estimation method of epicentral distance based on characteristics of P-wave initial envelope, RTRI Rept. 26, 5-10 (in Japanese).

[23] Nazeri, S., \& Z. H. Shomali (2019). Rapid estimation of the epicentral distance in the earthquake early warning system around the Tehran region, Iran, Seismol. Res. Lett. 90, no. 5, 1916-1922, doi: 10.1785/0220180375.

[24] Colombelli, S., Festa, G., \& Zollo, A. (2020). Early rupture signals predict the final earthquake size. Geophys. J. Int. 223 (1), 692-706. doi:10.1093/gji/ggaa343.

[25] Mjaavatten, A. (2021). Curvature of a 1D curve in a 2D or 3D space (https://www.mathworks.com/matlabcentral/fileexchange /69452-curvature-of-a-1d-curve-in-a-2d-or-3d-space), MATLAB Central File Exchange. Retrieved June 29, 2021.

[26] Kjartansson, E. (1979). Constant Q-wave propagation and attenuation. Journal of Geophysical Research, 84, 47374748.

[27] Abercrombie, R. (1996). The magnitude-frequency distribution of earthquakes recorded with deep 
seismometers at Cajon Pass, southern California, Tectonophysics, 261, 1-7.

[28] Courboulex, F., Virieux, J., Deschamps, A., Gibert, D., \& Zollo, A. (1996). Source investigation of a small event using empirical Green's functions and simulated annealing. Geophysical Journal International, 125, 768780 , https://doi.org/10.1111/j.1365-

246X.1996.tb06022.x.

[29] CREWES, 2020, Abstract Book: CREWES Research Report, 32, 86.

[30] Oth A. Bindi D. Parolai S., \& Di Giacomo D. (2010). Earthquake scaling characteristics and the scale(in)dependence of seismic energy-to-moment ratio: Insights from KiK-net data in Japan, Geophys. Res. Lett. 37, no. 19, L19304, doi: https://doi.org/10.1029/2010GL044572.

[31] Oth, A. (2013). On the characteristics of earthquake stress release variations in Japan, Earth and Planetary Science Letters, 377-378, 132-141, https://doi.org/10.1016/j.epsl.2013.06.037.

[32] Chiarabba, C., Piccinini, D., \& De Gori, P. (2009). Velocity and attenuation tomography of the Umbria Marche 1997 fault system: Evidence of a fluid-governed seismic sequence. Tectonophysics, 476 (1-2), 73-84, https://doi.org/10.1016/j.tecto.2009.04.004.

[33] De Lorenzo, S., Zollo, A., \& Zito, G. (2010). Source, attenuation, and site parameters of the 1997 Umbria Marche seismic sequence from the inversion of $P$ wave spectra: a comparison between constant $\mathrm{QP}$ and frequency dependent QP models. J. geophys. Res.115, doi:10.1029/2009JB007004.

[34] Wang, Z., \& Zhao, D. (2019). Updated attenuation tomography of Japan subduction zone, Geophysical Journal International, $219 \quad$ (3), 16791697, https://doi.org/10.1093/gji/ggz339

[35] Brune, J. N. (1971). Correction, J. Geophys. Res., 76, pp. 5002.

[36] Allmann, B.P. \& Shearer, P.M., (2009). Global variations of stress drop for moderate to large earthquakes, J. geophys. Res., 114, B01310, doi:10.1029/2008JB005821.

[37] Hasegawa A, Nakajima J, Uchida N, et al. (2009(. Plate subduction, and generation of earthquakes and magmas in Japan as inferred from seismic observations: an overview, Gondwana Research, 16(3-4): 370-400.

[38] Bindi, D., Cotton, F., Spallarossa, D., Picozzi, M., \& Rivalta, E. (2018). Temporal Variability of Ground Shaking and Stress Drop in Central Italy: A Hint for Fault Healing?. Bulletin of the Seismological Society of America, 108 (4): 1853-1863. doi: https://doi.org/10.1785/0120180078.

[39] Wang H, Ren Y, Wen R, Xu P. (2019). Breakdown of earthquake self-similar scaling and source rupture directivity in the 2016-2017 central Italy seismic sequence. J. Geophys. Res. Solid Earth 124, 3898-3917. (doi:10.1029/2018JB016543).

[40] Cheloni, D., De Novellis, V., Albano, M., et al. (2017). Geodetic model of the 2016 Central Italy earthquake sequence inferred from InSAR and GPS data: Modeling 2016 Central Italy Earthquakes. Geophys. Res. Lett. 44, 6778-6787. https://doi.org/10.1002/2017GL073580.

[41] Xu, G., Xu, C., Wen, Y., Jiang, G., 2017. Source Parameters of the 2016-2017 Central Italy Earthquake Sequence from the Sentinel-1, ALOS-2 and GPS Data. Remote Sensing 9, 1182. https://doi.org/10.3390/rs9111182.

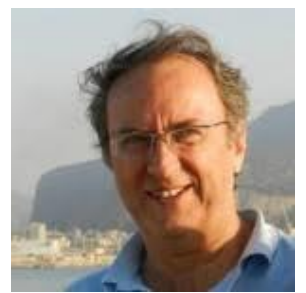

Aldo Zollo is full professor of Seismology and Signal Analysis and Processing at the University of Naples "Federico II". In his research, he deals with the theoretical and experimental aspects of the propagation of seismic waves and fracture processes in the Earth's crust. He was the PI of numerous research projects funded by national and international bodies in the areas of $1 /$ Seismic exploration of volcanoes, $2 /$ the modeling of source processes and $3 /$ the development of early warning systems for seismic prevention. He was, Member of the National Commission for Major Risks Forecast and Prevention appointed by the President of the Council of Ministers, of the Scientific Councils of INGV (Italy)and of the MISE Commission on the preparation of guidelines for monitoring induced seismicity in areas of hydrocarbon extraction and cultivation.

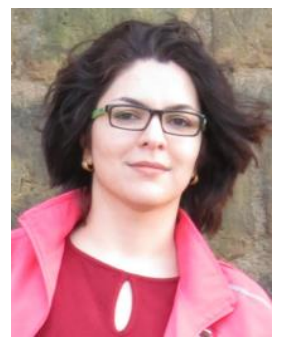

Sahar Nazeri received the bachelor's degrees in Physics from Razi University, Kermanshah, Iran, and two master's degrees in Physics, Condensed Matter and Geophysics, Earthquake Seismology from Razi University and University of Tehran, respectively. She received her Ph.D. degree from Naples Federico II University, Italy, in 2020, and she is currently working as a postdoc researcher in the same university. Her research interests include modelling of the earthquake source processes, signal processing, and earthquake early warning as well.

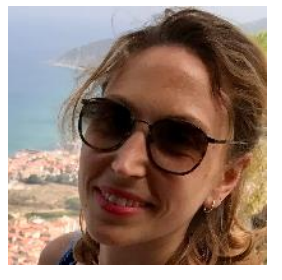

Simona Colombelli Simona Colombelli is a researcher at the Department of Physics of the University of Naples, Federico II. She has been exploring both the practical aspects of the implementation of warning systems and the theoretical issues related to the physics of the rupture process. She is currently the PI of a research project on the development of innovative methodologies and technologies for the real-time monitoring of earthquakes, funded by the European Community and by the Italian Ministry of Education. 
She is also the PI of a research project funded by the University of Naples Federico II, aimed at the understanding and modeling of physical processes of seismic ruptures. For her experience on Earthquake Early Warning, she has been co-convener of a dedicated session at the EGU General Assembly 2014, in Vienna and has been invited in 2016 as Guest Lecturer at the University of Aberdeen. 